\title{
On the Complete Join of Permutative Combinatorial Rees-Sushkevich Varieties
}

\author{
Edmond W. H. Lee \\ Nova Southeastern University, edmond.lee@nova.edu
}

Follow this and additional works at: https://nsuworks.nova.edu/math_facarticles

Part of the Mathematics Commons

\section{NSUWorks Citation}

Lee, Edmond W. H., "On the Complete Join of Permutative Combinatorial Rees-Sushkevich Varieties" (2007). Mathematics Faculty Articles. 139.

https://nsuworks.nova.edu/math_facarticles/139 
International Journal of Algebra, Vol. 1, 2007, no. 1, 1-9

\title{
On the Complete Join of Permutative Combinatorial Rees-Sushkevich Varieties
}

\author{
Edmond W. H. Lee ${ }^{1}$ \\ Department of Mathematics, Simon Fraser University \\ Burnaby, BC V5A 1S6, Canada \\ ewl@sfu.ca
}

\begin{abstract}
A semigroup variety is a Rees-Sushkevich variety if it is contained in a periodic variety generated by 0 -simple semigroups. The collection of all permutative combinatorial Rees-Sushkevich varieties constitutes an incomplete lattice that does not contain the complete join $\mathbf{J}$ of all its varieties. The objective of this article is to investigate the subvarieties of $\mathbf{J}$. It is shown that $\mathbf{J}$ is locally finite, non-finitely generated, and contains only finitely based subvarieties. The subvarieties of $\mathbf{J}$ are precisely the combinatorial Rees-Sushkevich varieties that do not contain a certain semigroup of order four.
\end{abstract}

Mathematics Subject Classification: 20M07, $08 \mathrm{~B} 15$.

Keywords: Semigroups, varieties, Rees-Sushkevich, permutative.

\section{Introduction}

Recall that a semigroup is 0 -simple if it does not contain any nontrivial proper ideals. The class of 0 -simple semigroups is one of the most important classes of semigroups. Indeed, as each finite semigroup can be obtained from finite 0 -simple semigroups by a sequence of ideal extensions, the role that finite 0 simple semigroups play in semigroup theory is comparable to the role that finite simple groups play in group theory. Naturally, the varieties generated by 0 -simple semigroups and their subvarieties deserve special attention.

Following Kublanovsky [4], any subvariety of a periodic variety generated by 0 -simple semigroups is said to be a Rees-Sushkevich variety. Investigation of the lattice of Rees-Sushkevich varieties has recently been initiated by Reilly, Volkov, and the author (see [5]-[10], [12]-[14], and [19]). In particular, several aspects of the lattice $\mathfrak{C}$ of combinatorial Rees-Sushkevich varieties have

\footnotetext{
${ }^{1}$ Research supported by NSERC of Canada, grant A4044.
} 
been considered in [5]-[7], [10], and [19]. Recall that a semigroup variety is combinatorial if all groups in it are trivial.

A semigroup variety is permutative if it satisfies some permutation identity. Denote by $\mathfrak{P}$ the set of all permutative varieties in $\mathfrak{C}$. It is easy to show that $\mathfrak{P}$ is a lattice (Proposition 5). However, the complete join $\mathbf{J}$ of all varieties in $\mathfrak{P}$ is not permutative (Proposition 11) so that $\mathfrak{P}$ is an incomplete sublattice of the subvariety lattice $\mathcal{L}(\mathbf{J})$ of $\mathbf{J}$. The structure of $\mathcal{L}(\mathbf{J})$ is quite complex, for it follows from a result of Vernikov and Volkov [17] that every finite lattice is embeddable in $\mathcal{L}(\mathbf{J})$.

The objective of the present article is to investigate the variety $\mathbf{J}$ and its subvarieties. Specifically, it is shown that the variety $\mathbf{J}$ is locally finite, nonfinitely generated, and contains only finitely based subvarieties. Consequently, $\mathcal{L}(\mathbf{J})$ is a countably infinite lattice. It is also shown that the subvarieties of $\mathbf{J}$ are precisely the varieties in $\mathfrak{C}$ that do not contain a certain semigroup of order four. The aforementioned properties of $\mathbf{J}$ are presented in Section 4.

\section{Background}

Let $X^{+}$and $X^{*}$ respectively be the free semigroup and free monoid over a countably infinite alphabet $X$. Elements of $X$ are referred to as letters, and elements of $X^{+}$and $X^{*}$ are referred to as words.

The head and tail of a word $\mathbf{u}$ are respectively the first and last letters occurring in $\mathbf{u}$ and are denoted by $\mathrm{h}(\mathbf{u})$ and $\mathrm{t}(\mathbf{u})$. The length of $\mathbf{u}$ is the number $|\mathbf{u}|$ of letters occurring in $\mathbf{u}$ counting multiplicity. The content of $\mathbf{u}$ is the set of letters occurring in $\mathbf{u}$ and is denoted by $\mathrm{C}(\mathbf{u})$. The set of length-two factors of $\mathbf{u}$ is $C_{2}(\mathbf{u})=\left\{\mathbf{w} \in X^{+}:|\mathbf{w}|=2\right.$ and $\left.\mathbf{u} \in X^{*} \mathbf{w} X^{*}\right\}$. It is easy to see that $\mathrm{C}_{2}(\mathbf{u})=\mathrm{C}_{2}(\mathbf{v})$ implies that $\mathrm{C}(\mathbf{u})=\mathrm{C}(\mathbf{v})$.

We write $\mathbf{u}=\mathbf{v}$ when $\mathbf{u}$ and $\mathbf{v}$ are identical words and write $\mathbf{u} \approx \mathbf{v}$ to stand for a semigroup identity. Let $\Sigma$ be a set of identities. We write $\Sigma \vdash \mathbf{u} \approx \mathbf{v}$ or

$\mathbf{u} \stackrel{\Sigma}{\approx} \mathbf{v}$ if the identity $\mathbf{u} \approx \mathbf{v}$ is derivable from the identities in $\Sigma$. The variety defined by $\Sigma$ is the class of all semigroups that satisfy all identities in $\Sigma$ and is denoted by $[\Sigma]$. If $\mathbf{V}$ is a variety with $\mathbf{V}=[\Sigma]$, then $\Sigma$ is said to be a basis for $\mathbf{V}$. A variety is finitely based if it possesses a finite basis.

A permutation identity is an identity of the form $x_{1} \cdots x_{m} \approx x_{1 \alpha} \cdots x_{m \alpha}$ where $x_{1}, \ldots, x_{m}$ are distinct letters and $\alpha$ is a nontrivial permutation on $\{1, \ldots, m\}$. A permutative variety is a variety that satisfies some permutation identity.

Lemma 1 (Perkins [11]) Each permutation identity implies the identity

$$
x_{1} \cdots x_{m} y z w_{1} \cdots w_{m} \approx x_{1} \cdots x_{m} z y w_{1} \cdots w_{m} \quad\left(\pi_{m}\right)
$$

for some $m \geq 1$. 
We refer the reader to [3] and [2] respectively for undefined terminology in semigroup theory and universal algebra.

\section{$3 \quad$ The subvariety lattice $\mathcal{L}\left(\mathbf{A}_{2}\right)$ of $\mathbf{A}_{2}$}

Recall that $\mathfrak{C}$ denotes the lattice of all combinatorial Rees-Sushkevich varieties and $\mathfrak{P}$ denotes the set of all permutative varieties in $\mathfrak{C}$.

Denote by $A_{2}$ the idempotent-generated 0-simple semigroup of order five and by $B_{2}$ the Brandt semigroup of order five:

$$
\begin{aligned}
& A_{2}=\left\langle a, b: a^{2}=a b a=a, b^{2}=0, b a b=b\right\rangle, \\
& B_{2}=\left\langle c, d: c^{2}=d^{2}=0, c d c=c, d c d=d\right\rangle .
\end{aligned}
$$

These two semigroups play very important roles in the theory of semigroup and especially in the theory of semigroup varieties. They appeared or were investigated in, for example, [1], [3]-[16], and [19]. Denote by $\mathbf{A}_{\mathbf{2}}$ the variety generated by $A_{2}$.

Proposition 2 ([10, Proposition 1.2]) The variety $\mathbf{A}_{\mathbf{2}}$ is the largest combinatorial Rees-Sushkevich variety. Consequently, the lattice $\mathfrak{C}$ coincides with the subvariety lattice $\mathcal{L}\left(\mathbf{A}_{\mathbf{2}}\right)$ of $\mathbf{A}_{\mathbf{2}}$.

In view of Proposition 2 , any variety $\mathbf{V}$ in $\mathfrak{C}$ is defined within $\mathbf{A}_{\mathbf{2}}$ by some set $\Sigma$ of identities, that is, $\mathbf{V}=\mathbf{A}_{\mathbf{2}} \cap[\Sigma]$.

Lemma 3 (Trahtman $[15,16]$ ) The identities

$$
x^{3} \approx x^{2}, \quad \text { xyxyx } \approx x y x, \quad \text { xyxzx } \approx x z x y x
$$

constitute a basis for $\mathbf{A}_{\mathbf{2}}$. More generally, an identity $\mathbf{u} \approx \mathbf{v}$ holds in $\mathbf{A}_{\mathbf{2}}$ if and only if $\mathrm{C}_{2}(\mathbf{u})=\mathrm{C}_{2}(\mathbf{v}), \mathrm{h}(\mathbf{u})=\mathrm{h}(\mathbf{v})$, and $\mathrm{t}(\mathbf{u})=\mathrm{t}(\mathbf{v})$.

A word of length at least two is said to be connected if it cannot be written as a product of two nonempty words with disjoint contents.

Lemma 4 Let $\mathbf{u}, \mathbf{v}$ be connected words such that $\mathrm{C}(\mathbf{u})=\mathrm{C}(\mathbf{v}), \mathrm{h}(\mathbf{u})=\mathrm{h}(\mathbf{v})$, and $\mathrm{t}(\mathbf{u})=\mathrm{t}(\mathbf{v})$. Suppose $\sigma$ is an identity that does not hold in the semigroup $B_{2}$. Then the identity $\mathbf{u} \approx \mathbf{v}$ holds in the variety $\mathbf{A}_{\mathbf{2}} \cap[\sigma]$. In particular, the following identities hold in any subvariety of $\mathbf{A}_{\mathbf{2}}$ that does not contain $B_{2}$ :

$$
\begin{aligned}
x p y z q x & \approx x p z y q x, x p y z x \\
x p y^{2} q x \approx x p z y x, x y z q x & \approx x z y q x, x, x p y^{2} x \approx x p y x, x y^{2} q x \approx x y q x, x y^{2} x \approx x y x \\
x^{2} y x & \approx x y x, x y x^{2} \approx x y x .
\end{aligned}
$$


Proof. Without loss of generality, suppose $\mathrm{C}(\mathbf{u})=\mathrm{C}(\mathbf{v})=\left\{x_{1}, \ldots, x_{n}\right\}$. Denote by $F_{n}$ the free object of $\mathbf{A}_{\mathbf{2}} \cap[\sigma]$ over the generators $\left\{x_{1}, \ldots, x_{n}\right\}$. Since $\mathbf{A}_{2}$ is locally finite, $F_{n}$ is a finite semigroup. It follows from [1, Exercise 8.1.6] that the regular $\mathcal{D}$-classes of $F_{n}$ are subsemigroups.

Now $\mathbf{u}$ and $\mathbf{v}$ are regular elements of $F_{n}$ by [10, Proposition 2.2], and it follows from [1, Theorem 8.1.7] that they belong to the same $\mathcal{D}$-class $D$, which must be a rectangular band. Since $C_{2}(\mathbf{u v})=C_{2}(\mathbf{v u}), h(\mathbf{u v})=h(\mathbf{v u})$, and $\mathrm{t}(\mathbf{u v})=\mathrm{t}(\mathbf{v u})$, the identity $\mathbf{u v} \approx \mathbf{v} \mathbf{u}$ holds in $\mathbf{A}_{\mathbf{2}}$ by Lemma 3. Therefore $\mathbf{u}$ and $\mathbf{v}$ are commuting elements in the rectangular band $D$ and so must coincide in $F_{n}$.

Proposition 5 The set $\mathfrak{P}$ constitutes a sublattice of $\mathcal{L}\left(\mathbf{A}_{\mathbf{2}}\right)$.

Proof. Suppose $\mathbf{U}, \mathbf{V} \in \mathfrak{P}$. Clearly $\mathbf{U} \cap \mathbf{V}$ is a variety in $\mathfrak{C}$ that satisfies all (permutation) identities of $\mathbf{U}$ and $\mathbf{V}$ so that $\mathbf{U} \cap \mathbf{V} \in \mathfrak{P}$. By Lemma 1, there exist $i, j \geq 1$ such that the permutation identities $\pi_{i}$ and $\pi_{j}$ hold in $\mathbf{U}$ and $\mathbf{V}$ respectively. Then $\mathbf{U} \vee \mathbf{V}$ is a variety in $\mathfrak{C}$ that satisfies the identity $\pi_{m}$ where $m=\max \{i, j\}$. Therefore $\mathbf{U} \vee \mathbf{V} \in \mathfrak{P}$.

Lemma 6 The identities

$$
\begin{aligned}
x^{2} y z w^{2} & \approx x^{2} z y w^{2}, \\
x^{2} y^{2} z^{2} & \approx x^{2} y z^{2},
\end{aligned}
$$

define the same subvariety in $\mathbf{A}_{\mathbf{2}}$, that is, $\mathbf{A}_{\mathbf{2}} \cap[(5 \mathrm{a})]=\mathbf{A}_{\mathbf{2}} \cap[(5 \mathrm{~b})]$.

Proof. It is straightforward to show that the identities (5a) and (5b) do not hold in $B_{2}$. Hence $\{(1),(5 \mathrm{a})\} \vdash(3)$ and $\{(1),(5 \mathrm{~b})\} \vdash(2)$ by Lemma 4 . It follows that the inclusion $\mathbf{A}_{\mathbf{2}} \cap[(5 \mathrm{a})] \subseteq \mathbf{A}_{\mathbf{2}} \cap[(5 \mathrm{~b})]$ holds since

$$
\begin{aligned}
\{(1),(5 \mathrm{a})\} \vdash x^{2} y^{2} z^{2} & \stackrel{(1)}{\approx} x^{2} x y^{2} z^{2} \stackrel{(5 \mathrm{a})}{\approx} x^{2} y^{2} x z^{2} \\
& \stackrel{(3)}{\approx} x^{2} y x z^{2} \stackrel{(5 \mathrm{a})}{\approx} x^{2} x y z^{2} \\
& \stackrel{(1)}{\approx} x^{2} y z^{2}
\end{aligned}
$$

and the inclusion $\mathbf{A}_{\mathbf{2}} \cap[(5 \mathrm{~b})] \subseteq \mathbf{A}_{\mathbf{2}} \cap[(5 \mathrm{a})]$ holds since

$$
\begin{aligned}
\{(1),(5 \mathrm{~b})\} \vdash x^{2} y z w^{2} & \stackrel{(1)}{\approx} x^{2}(x y z w) w^{2} \stackrel{(5 \mathrm{~b})}{\approx} x^{2}(x y z w)^{2} w^{2} \\
& \stackrel{(2)}{\approx} x^{2}(x z y w)^{2} w^{2} \stackrel{(5 \mathrm{~b})}{\approx} x^{2} x z y w w^{2} \\
& \stackrel{(1)}{\approx} x^{2} z y w^{2} .
\end{aligned}
$$


Note that by Lemma 4, all subvarieties of $\mathbf{A}_{\mathbf{2}} \cap[(5 \mathrm{a})]$ satisfy the identities (2), (3), and (4). This result will be used in the remainder of this article without further reference.

A word is simple if all letters occurring in it have multiplicity one. Suppose $X$ is (alphabetically) ordered by $<$. A word $\mathbf{u}=x_{1} \cdots x_{m}$ is said to be an ordered word if $x_{1}<\cdots<x_{m}$. Clearly an ordered word is necessarily simple. A word $\mathbf{u}$ is said to be in canonical form if any of the following conditions hold:

(A) $\mathbf{u}=x \mathbf{v} x$ for some ordered word $\mathbf{v} \in X^{*}$ with $x \notin \mathrm{C}(\mathbf{v})$;

(B) $\mathbf{u}=x y \mathbf{v} x y$ for some ordered word $\mathbf{v} \in X^{*}$ with $x, y \notin \mathrm{C}(\mathbf{v})$ and $x \neq y$.

Note that a word in canonical form is necessarily connected.

Lemma 7 Let $\mathbf{u}$ be a connected word. Then there exists a unique word $\mathbf{u}^{\star}$ in canonical form such that $\mathrm{C}(\mathbf{u})=\mathrm{C}\left(\mathbf{u}^{\star}\right), \mathrm{h}(\mathbf{u})=\mathrm{h}\left(\mathbf{u}^{\star}\right)$, and $\mathrm{t}(\mathbf{u})=\mathrm{t}\left(\mathbf{u}^{\star}\right)$. Further, the identity $\mathbf{u} \approx \mathbf{u}^{\star}$ holds in the variety $\mathbf{A}_{\mathbf{2}} \cap[(5 \mathrm{a})]$.

Proof. The existence and uniqueness of $\mathbf{u}^{\star}$ is easy to verify. Since any word in canonical form is connected, the identity $\mathbf{u} \approx \mathbf{u}^{\star}$ holds in $\mathbf{A}_{\mathbf{2}} \cap[(5 \mathrm{a})]$ by Lemma 4 .

Lemma 8 A non-simple word $\mathbf{u}$ is equivalent within $\mathbf{A}_{\mathbf{2}} \cap[(5 \mathrm{a})]$ to a word $\mathbf{p w}^{\star} \mathbf{q}$ where

(i) $\mathbf{p}, \mathbf{q} \in X^{*}$ are simple words;

(ii) $\mathbf{w} \in X^{+}$is a connected word;

(iii) $\mathrm{C}(\mathbf{p}), \mathrm{C}(\mathbf{w}), \mathrm{C}(\mathbf{q})$ are pairwise disjoint sets.

Proof. By assumption, we may write $\mathbf{u}=\mathbf{p v q}$ where $\mathbf{p}, \mathbf{q} \in X^{*}$ are simple, $\mathbf{v} \in X^{+}$is non-simple with $h=\mathrm{h}(\mathbf{v})$ and $t=\mathrm{t}(\mathbf{v})$ each occurring at least twice in $\mathbf{v}$, and $\mathbf{C}(\mathbf{p}), \mathbf{C}(\mathbf{v}), \mathbf{C}(\mathbf{q})$ are pairwise disjoint sets. (Note that $h=t$ is possible). The words $\mathbf{v}$ and $\mathbf{v}^{2}$ are equivalent within $\mathbf{A}_{\mathbf{2}} \cap[(5 \mathrm{a})]$ since

$$
\mathbf{v} \stackrel{(4)}{\approx} h^{2} \mathbf{v} t^{2} \stackrel{(5 \mathrm{~b})}{\approx} h^{2} \mathbf{v}^{2} t^{2} \stackrel{(4)}{\approx} \mathbf{v}^{2}
$$

Hence, by Lemma 7 , the words $\mathbf{u}$ and $\mathbf{p} \mathbf{w}^{\star} \mathbf{q}$ are equivalent within $\mathbf{A}_{\mathbf{2}} \cap[(5 \mathrm{a})]$ where the word $\mathbf{w}=\mathbf{v}^{2}$ is connected.

Proposition 9 Every subvariety of $\mathbf{A}_{2} \cap[(5 \mathrm{a})]$ is finitely based.

Proof. The variety $\mathbf{A}_{\mathbf{2}} \cap[(5 \mathrm{a})]$ is clearly finitely based. Suppose $\mathbf{V}$ is a proper subvariety of $\mathbf{A}_{\mathbf{2}} \cap[(5 \mathrm{a})]$. Then $\mathbf{V}$ is defined within $\mathbf{A}_{\mathbf{2}} \cap[(5 \mathrm{a})]$ by some set $\Sigma$ of identities. By Lemma 8, we may assume that all identities in $\Sigma$ are formed by words that are either simple or of the form $\mathbf{p w}^{\star} \mathbf{q}$. It follows from [18] that $\mathbf{V}$ is finitely based. 


\section{Main results}

Recall that $\mathbf{J}$ denotes the complete join of all varieties in $\mathfrak{P}$. This section presents several properties of $\mathbf{J}$ and its subvarieties.

Proposition $10 \quad \mathbf{J}=\mathbf{A}_{\mathbf{2}} \cap[(5 \mathrm{a})]$.

Proof. It is easy to see that within $\mathbf{A}_{\mathbf{2}}$, the identity (5a) is a consequence of the identity $\pi_{m}$ for any $m \geq 1$. Therefore $\mathbf{J} \subseteq \mathbf{A}_{\mathbf{2}} \cap[(5 \mathrm{a})]$.

Suppose $\mathbf{V}$ is a variety such that $\mathbf{J} \subset \mathbf{V} \subset \mathbf{A}_{\mathbf{2}} \cap[(5 \mathrm{a})]$. By Proposition 9, $\mathbf{V}$ is defined within $\mathbf{A}_{\mathbf{2}} \cap[(5 \mathrm{a})]$ by some finite set $\Sigma$ of identities. We may assume that the identities in $\Sigma$ do not hold in $\mathbf{A}_{\mathbf{2}} \cap[(5 \mathrm{a})]$. By Lemma 8, we may assume that all identities in $\Sigma$ are formed by words that are either simple or of the form $\mathbf{p w}^{\star} \mathbf{q}$. Further, since $\mathbf{J}$ contains semilattices, each identity in $\Sigma$ is formed by a pair of words with identical content. Let $\sigma: \mathbf{u} \approx \mathbf{v}$ be an identity in $\Sigma$.

Case 1: Suppose both $\mathbf{u}$ and $\mathbf{v}$ are simple. Then $\sigma$ is a permutation identity. It follows that $\mathbf{V} \subseteq \mathbf{A}_{\mathbf{2}} \cap[\sigma] \subseteq \mathbf{J}$, which is a contradiction.

Case 2: Suppose $\mathbf{u}$ is simple and $\mathbf{v}$ is non-simple (of the form $\mathbf{p w}^{\star} \mathbf{q}$ ). Then $\mathbf{u}=x_{1} \cdots x_{m}$ and $\mathbf{v}=\mathbf{v}_{1} x_{k} \mathbf{v}_{2} x_{k} \mathbf{v}_{3}$ for some $k \in\{1, \ldots, m\}$ and some $\mathbf{v}_{1}, \mathbf{v}_{2}, \mathbf{v}_{3} \in X^{*}$ with $\mathrm{C}\left(\mathbf{v}_{1} \mathbf{v}_{2} \mathbf{v}_{3}\right)=\mathrm{C}(\mathbf{u}) \backslash\left\{x_{k}\right\}$. Denote by $\varphi$ the substitution $x_{k} \mapsto x y z w$ and by $\chi$ the substitution $x_{k} \mapsto x z y w$. Then

$$
\begin{aligned}
\{(1),(2), \sigma\} \vdash \mathbf{u} \varphi & \stackrel{\sigma}{\approx} \mathbf{v} \varphi \\
& =\mathbf{v}_{1} x y z w \mathbf{v}_{2} x y z w \mathbf{v}_{3} \\
& \stackrel{(2)}{\approx} \mathbf{v}_{1} x z y w \mathbf{v}_{2} x z y w \mathbf{v}_{3} \\
& =\mathbf{v} \chi \stackrel{\sigma}{\approx} \mathbf{u} \chi .
\end{aligned}
$$

Hence $\mathbf{V}$ satisfies the permutation identity $\mathbf{u} \varphi \approx \mathbf{u} \chi$, and we arrive at the same contradiction in Case 1.

Therefore Cases 1 and 2 are both impossible, whence all identities in $\Sigma$ are formed by non-simple words of the form $\mathbf{p w}^{\star} \mathbf{q}$. Suppose $\tau: \mathbf{u}_{1} \approx \mathbf{u}_{2}$ is such an identity in $\Sigma$, say $\mathbf{u}_{i}=\mathbf{p}_{i} \mathbf{w}_{i}^{\star} \mathbf{q}_{i}$ for $i \in\{1,2\}$. It is easy to show that if

$$
\mathbf{p}_{1}=\mathbf{p}_{2}, \quad \mathbf{q}_{1}=\mathbf{q}_{2}, \quad \mathrm{~h}\left(\mathbf{w}_{1}\right)=\mathrm{h}\left(\mathbf{w}_{2}\right), \quad \text { and } \quad \mathrm{t}\left(\mathbf{w}_{1}\right)=\mathrm{t}\left(\mathbf{w}_{2}\right),
$$

then $\mathbf{u}_{1}=\mathbf{u}_{2}$ so that the identity $\tau$ is contradictorily satisfied by $\mathbf{A}_{\mathbf{2}} \cap[(5 \mathrm{a})]$. Thus at least one of the four equalities in (6) do not hold, whence $\left\{(1), \pi_{m}\right\} \nvdash \tau$ for any $m \geq \max \left\{\left|\mathbf{u}_{1}\right|,\left|\mathbf{u}_{2}\right|\right\}$. But this contradicts the fact that $\mathbf{A}_{\mathbf{2}} \cap\left[\pi_{m}\right] \subseteq$ $\mathbf{J} \subset \mathbf{V} \subseteq \mathbf{A}_{\mathbf{2}} \cap[\tau]$. Consequently, the identity $\tau$, and hence $\mathbf{V}$, do not exist, whence $\mathbf{J}=\mathbf{A}_{\mathbf{2}} \cap[(5 \mathrm{a})]$. 
Proposition 11 The variety $\mathbf{J}$ is not permutative. Consequently, the lattice $\mathfrak{P}$ is incomplete.

Proof. By referring to the identity basis for $\mathbf{J}$ in Proposition 10, it is easy to show that $\mathbf{J}$ does not satisfy any of the identities $\pi_{m}$ and hence cannot be permutative by Lemma 1 . Therefore $\mathfrak{P}$ does not contain the complete join $\mathbf{J}$ of its varieties, whence it is a lattice (Proposition 5) that is incomplete.

Proposition 12 The variety $\mathbf{J}$ is locally finite and non-finitely generated.

Proof. The variety $\mathbf{J}$ is locally finite since $\mathbf{A}_{\mathbf{2}}$ is finitely generated. Let $S$ be a semigroup in $\mathbf{J}$ with $|S|<m$. For $1 \leq i \leq m$, let $a, b, g_{i}, h_{i} \in S$. Since the list $g_{1}, \ldots, g_{m}$ contains an element (say $g_{i}$ ) that appears at least twice and that $S$ satisfies the identities (4), we have

$$
g_{1} \cdots g_{m} \stackrel{(4)}{=} g_{1} \cdots g_{i-1} g_{i}^{2} g_{i} \cdots g_{m}
$$

By an identical argument, $h_{1} \cdots h_{m} \stackrel{(4)}{=} h_{1} \cdots h_{j} h_{j}^{2} h_{j+1} \cdots h_{m}$ for some $j$. The identities (2) and (5b) also hold in $S$ so that

$$
\begin{aligned}
& g_{1} \cdots g_{m} \cdot a b \cdot h_{1} \cdots h_{m} \\
& \stackrel{(4)}{=} g_{1} \cdots g_{i-1}\left(g_{i}^{2} g_{i} \cdots g_{m} \cdot a b \cdot h_{1} \cdots h_{j} h_{j}^{2}\right) h_{j+1} \cdots h_{m} \\
& \stackrel{(5 \mathrm{~b})}{=} g_{1} \cdots g_{i-1} g_{i}^{2}\left(g_{i} \cdots g_{m} \cdot a b \cdot h_{1} \cdots h_{j}\right)^{2} h_{j}^{2} h_{j+1} \cdots h_{m} \\
& \stackrel{(2)}{=} g_{1} \cdots g_{i-1} g_{i}^{2}\left(g_{i} \cdots g_{m} \cdot b a \cdot h_{1} \cdots h_{j}\right)^{2} h_{j}^{2} h_{j+1} \cdots h_{m} \\
& \stackrel{(5 \mathrm{~b})}{=} g_{1} \cdots g_{i-1} g_{i}^{2} g_{i} \cdots g_{m} \cdot b a \cdot h_{1} \cdots h_{j} h_{j}^{2} h_{j+1} \cdots h_{m} \\
& \stackrel{(4)}{=} g_{1} \cdots g_{m} \cdot b a \cdot h_{1} \cdots h_{m} .
\end{aligned}
$$

Hence $S$ satisfies the identity $\pi_{m}$. But $\mathbf{J}$ does not satisfy $\pi_{m}$ (Proposition 11) and so cannot be generated by $S$.

Proposition 13 Every subvariety of $\mathbf{J}$ is finitely based. Consequently, $\mathcal{L}(\mathbf{J})$ is a countably infinite lattice.

Proof. The first part follows from Propositions 9 and 10, while the second part holds since only countably many finite sets of identities exist up to relabelling of letters.

The last result of this article involves the semigroup

$$
Y=\left\langle e, f, s: e^{2}=e, f^{2}=f, e f=f e=0, e s=s f=s\right\rangle
$$

of order four. It is easy to show that $Y$ is isomorphic to a subsemigroup of the 0-simple semigroup $B_{2}$ and so belongs to $\mathbf{A}_{\mathbf{2}}$ by Proposition 2. 
Theorem 14 The following statements on a variety $\mathbf{V}$ in $\mathfrak{C}$ are equivalent.

(i) $\mathbf{V}$ is contained in $\mathbf{J}$;

(ii) $\mathbf{V}$ does not contain $Y$.

Consequently, $\mathbf{J}$ is the largest variety in $\mathfrak{C}$ that does not contain $Y$.

Proof. Since $e^{2} s e f^{2}=0 \neq s=e^{2} e s f^{2}$, the identity (5a) of $\mathbf{J}$ does not hold in the semigroup $Y$ so that statement (i) implies statement (ii).

Conversely, suppose statement (ii) holds. Let $S$ be any finite semigroup in V. It follows from [1, Proposition 11.8.1] and the identities (1) of $\mathbf{A}_{\mathbf{2}}$ that $S$ satisfies at least one of the identities

$$
\begin{aligned}
\left(x^{2} y z^{2}\right)^{2} & \approx x^{2} y z^{2}, \\
x^{2} y z^{2} x^{2} z^{2} & \approx x^{2} y z^{2}, \\
x^{2} z^{2} x^{2} y z^{2} & \approx x^{2} y z^{2} .
\end{aligned}
$$

The variety generated by $S$ does not contain $B_{2}$ so that $S$ also satisfies the identities (3) by Lemma 4 . Note that

$$
\begin{aligned}
& \left\{(1),(3), \rho_{1}\right\} \vdash x^{2} y z^{2} \stackrel{\rho_{1}}{\approx}\left(x^{2} y z^{2}\right)^{2} \stackrel{(3)}{\approx}\left(x^{2} y^{2} z^{2}\right)^{2} \stackrel{\rho_{1}}{\approx} x^{2} y^{2} z^{2} \\
& \left\{(1),(3), \rho_{2}\right\} \vdash x^{2} y z^{2} \stackrel{\rho_{2}}{\approx} x^{2} y z^{2} x^{2} z^{2} \stackrel{(3)}{\approx} x^{2} y^{2} z^{2} x^{2} z^{2} \stackrel{\rho_{2}}{\approx} x^{2} y^{2} z^{2},
\end{aligned}
$$

and by a symmetrical argument, $\left\{(1),(3), \rho_{3}\right\} \vdash x^{2} y z^{2} \approx x^{2} y^{2} z^{2}$. Therefore the identity (5b) holds in $S$.

We have thus shown that the identity (5b) holds in every finite semigroup of the locally finite variety $\mathbf{V}$ and hence must also hold in $\mathbf{V}$. Consequently, $\mathbf{V} \subseteq \mathbf{A}_{\mathbf{2}} \cap[(5 \mathrm{~b})]=\mathbf{J}$ by Lemma 6 and Proposition 10.

\section{References}

[1] J. Almeida, Finite Semigroups and Universal Algebra, World Scientific, Singapore, 1994.

[2] S. Burris, H. P. Sankappanavar, A Course in Universal Algebra, Springer Verlag, New York, 1981.

[3] J. M. Howie, Fundamentals of Semigroup Theory, Clarendon Press, Oxford, 1995.

[4] S. I. Kublanovsky, On the Rees-Sushkevich variety, preprint.

[5] E. W.H. Lee, On the Lattice of Rees-Sushkevich Varieties, Ph.D. thesis, Simon Fraser University, 2002. 
[6] E. W. H. Lee, Identity bases for some non-exact varieties, Semigroup Forum, 68 (2004), 445-457.

[7] E. W. H. Lee, Subvarieties of the variety generated by the five-element Brandt semigroup, Internat. J. Algebra Comput., 16 (2006), 417-441.

[8] E. W. H. Lee, N. R. Reilly, Centrality in Rees-Sushkevich varieties, preprint.

[9] E. W.H. Lee, N.R. Reilly, The intersection of pseudovarieties of central simple semigroups, Semigroup Forum, to appear.

[10] E. W. H. Lee, M. V. Volkov, On the structure of the lattice of combinatorial Rees-Sushkevich varieties, Proceedings of the International Conference on Semigroups and Languages (Lisbon 2005), World Scientific, Singapore, to appear.

[11] P. Perkins, Bases for equational theories of semigroups, J. Algebra, 11 (1969), 298-314.

[12] N. R. Reilly, Complete congruences on the lattice of Rees-Sushkevich varieties, preprint.

[13] N. R. Reilly, Shades of orthodoxy in Rees-Sushkevich varieties, preprint.

[14] N. R. Reilly, Varieties generated by completely 0-simple semigroups, preprint.

[15] A. N. Trahtman, Graphs of identities of a completely 0-simple five-element semigroup, Ural Polytechnic Institute, Sverdlovsk, 1981, 6 pp. [Russian] (Deposited at VINITI, Moscow, no. 5558-81).

[16] A. N. Trahtman, Identities of a five-element 0-simple semigroup, Semigroup Forum, 48 (1994), 385-387.

[17] B. M. Vernikov and M. V. Volkov, Lattices of nilpotent varieties of semigroups. II, Izv. Ural. Gos. Univ. Mat. Mekh. No.1(10), (1998), 13-33. [Russian]

[18] M. V. Volkov, Finite basis theorem for systems of semigroup identities, Semigroup Forum, 28 (1984), 93-99.

[19] M. V. Volkov, On a question by Edmond W. H. Lee, Izv. Ural. Gos. Univ. Mat. Mekh. No.7(36), (2005), 167-178.

\section{Received: July 5, 2006}

